\title{
Mathematical Approach to Complexity-Reduced Antenna Selection Technique for Achieving High Channel Capacity
}

\author{
Priya Dhawan \\ Department of ECE \\ Amritsar College of Engineering and Technology \\ Amritsar-143001,Punjab,India
}

\author{
Narinder Sharma \\ Department of EEE \\ Amritsar College of Engineering and Technology \\ Amritsar-143001,Punjab,India
}

\begin{abstract}
In this paper channel state information is exploited for improving system performance. The performance parameters of the Multiple Input Multiple Output system is better and are even achieved using additional RF modules that are required as multiple antennas are employed. To reduce the cost associated with the multiple RF modules, antenna selection techniques can be used to employ a smaller number of RF modules than the number of transmit antennas. The exploiting of information for complexity reduced antenna selection is performed for achieving high channel capacity. Simulation results show that the channel capacity increases in proportion to the number of the selected antennas.
\end{abstract}

Keywords: MIMO systems, RF modules, Antenna Selection, Channel State Information, Signal to Noise Ratio.

\section{INTRODUCTION}

In typical digital communication system, Signal parameters on which multipath channel have effect that are independent path gain, independent path frequency offset, independent path phase shift, independent path time delay etc. To remove ISI from the signal, many kinds of equalizers can be used. Different techniques are used to handle the changes made by the channel,receiver requires knowledge over CIR to combat with the received signal for recovering the transmitted signal. CIR is provided by the separate channel estimator. Usually channel estimation is based on the known sequence of bits, which is unique for a certain transmitter and is repeated in every transmission burst. Which enables the channel estimator to estimate CIR for each burst separately by using the known transmitted signal and the corresponding received signal. Multiple Input Multiple Output (MIMO) systems takes advantage of multipath propagation signals by sending and receiving more than one data signal in the same frequency band at the same time by using multiple transmit and receive antennas. Orthogonal frequency division multiplexing (OFDM) is also has capability to handle the effect of ISI and Inter carrier interference (ICI). OFDM converts the frequency selective wide band signal into frequency flat multiple orthogonally spaced narrow band signals also resulting in high bandwidth efficiency [1].

\section{ANTENNA SELECTION TECHNIQUE}

The antenna selection technique is one of the major issue that is to be taken care in the communication system. MIMO systems have better performance which can be achieved without using additional transmit power or bandwidth extension.[2] However, it requires additional high-cost RF modules are required as multiple antennas are employed. In general, a transmitter does not have direct access to its own channel state information. Therefore, some indirect means are required for the transmitter. In time division duplexing system, we can exploit the channel reciprocity between opposite links (downlink and uplink). Based on the signal received from the opposite direction, it allows for indirect channel estimation. In frequency division duplexing (FDD) system, which usually does not have reciprocity between opposite directions, the transmitter relies on the channel feedback information from the receiver. In other words, CSI must be estimated at the receiver side and then, fed back to the transmitter side. To reduce the cost associated with the multiple RF modules, antenna selection techniques can be used to employ a smaller number of RF modules than the number of transmit antennas. Figure 1 illustrates the end-toend configuration of the antenna selection in which only Q RF modules are used to support $\mathrm{N}_{\mathrm{T}}$ transmit antennas since $\mathrm{Q} R \mathrm{RF}$ modules are selectively mapped to $\mathrm{Q}$ of $\mathrm{N}_{\mathrm{T}}$ transmit antennas.[2]

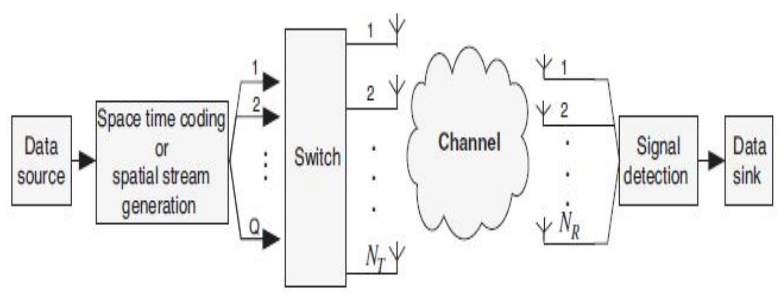

Figure 1: Antenna selections with $\mathbf{Q} \mathbf{R F}$ modules and $\mathbf{N}_{\mathbf{T}}$ transmit antennas $\left(Q<N_{T}\right)[10]$

Since $\mathrm{Q}$ antennas are used among $\mathrm{N}_{\mathrm{T}}$ transmit antennas, the effective channel can now be represented by $\mathrm{Q}$ columns of 
$H \in \mathfrak{R}^{N_{R} \times N_{T}}$. Let $p$ denote the index of the $\mathrm{i}^{\text {th }}$ selected column, $i=1,2, \cdots, Q$. Then, the corresponding effective channel will be modeled by $N_{R} \times Q$ matrix, which is denoted by $H_{\left\{p_{1}, p_{2}, \cdots p_{Q}\right\}} \in \mathfrak{R}^{N_{R} \times Q}$ [3]. Let $X \in \mathfrak{R}^{Q \times 1}$ denote the space-time-coded or spatially-multiplexed stream that is mapped into Q selected antennas. Then, the received signal $\mathrm{y}$ is represented as

$$
y=\sqrt{\frac{E_{X}}{Q}} H_{\left\{p_{1}, p_{2}, \cdots p_{Q}\right\}} X+Z
$$

where $z \in \mathfrak{R}^{N_{R} \times 1}$ is the additive noise vector. The channel capacity of the system in Equation (1) will depend on the number of transmit antennas that are chosen.

\section{COMPLEXITY-REDUCED ANTENNA SELECTION TECHNIQUE}

The Complexity-Reduced Antenna Selection Technique is one of the type of antenna selection technique. As compared to the optimal antenna technique, complexity reduced antenna selection technique is better. Optimal antenna selection requires too much complexity depending on the total number of available transmit antennas. In order to reduce its complexity, we proposed a sub-optimal method. We adopted an approach in which additional antenna is selected in ascending order of increasing the channel capacity i.e., one antenna with the highest capacity is first selected as

$$
\begin{aligned}
& p_{1}^{\text {subopt }}=\arg \max _{p_{1}} C_{\left\{p_{1}\right\}} \\
& =\arg \max _{p_{1}} \log _{2} \operatorname{det}\left(I_{N_{R}}+\frac{E_{X}}{Q N_{0}} H_{\left\{p_{1}\right\}} H_{\left\{p_{1}\right\}}^{H}\right)
\end{aligned}
$$

Given the first selected antenna, the second antenna is selected such that the channel capacity is maximized i.e.

$$
p_{2}^{\text {subopt }}=\underset{p_{2} \neq p_{1}^{\text {subopt }}}{\arg \max } C_{\left\{p_{1}^{\text {subpot }}, p_{2}\right\}}
$$

$$
\begin{gathered}
\underset{p_{2} \neq p_{1}^{\text {subopt }}}{\arg \max } \log _{2} \operatorname{det}\left(I_{N_{R}}+\frac{E_{X}}{Q N_{0}} H_{\left\{p_{1}^{\text {subpot }}, p_{2}\right\}}\right) \\
\text { After the nth iteration which } \\
\text { provides }\left\{p_{1}^{\text {subopt }}, p_{2}^{\text {subopt }}, \cdots p_{n}^{\text {subopt }}\right\} \text {, the capacity with }
\end{gathered}
$$

an additional antenna, say antenna 1 , can be updated as

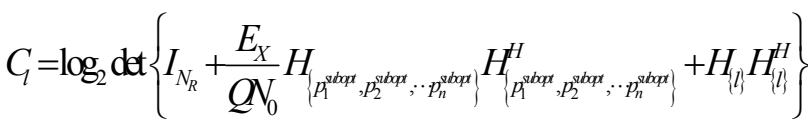

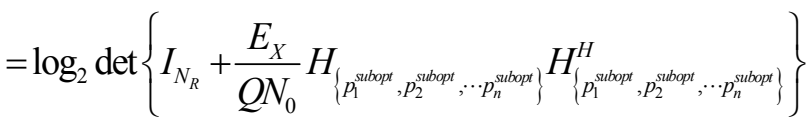

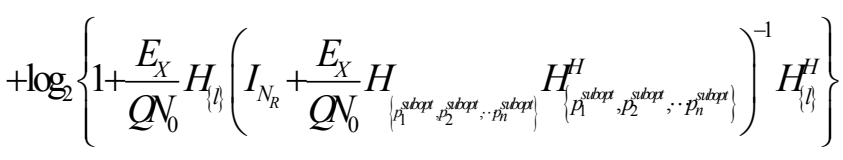

It can be derived using the following identities:

$$
\begin{aligned}
& \operatorname{det}\left(A+u v^{H}\right)=\left(1+V^{H} A^{-1} u\right) \operatorname{det}(A) \\
& \log _{2} \operatorname{det}\left(A+u v^{H}\right)=\log _{2}\left(1+V^{H} A^{-1} u\right) \operatorname{det}(A)+\log _{2}\left(1+V^{H} A^{-1} u\right)
\end{aligned}
$$

Where

$$
\begin{aligned}
& A=I_{N_{R}}+\frac{E_{X}}{Q N_{0}} H_{\left\{p_{1}^{\text {subopt }}, p_{2}^{\text {subopt }}, \cdots p_{n}^{\text {subop } t}\right\}} H_{\left\{p_{1}^{\text {subopt }}, p_{2}^{\text {subopt }}, \cdots p_{n}^{\text {subopt }}\right\}}^{H} \\
& u=v=\sqrt{\frac{E_{X}}{Q N_{0}}} H_{\{l\}}
\end{aligned}
$$

The additional $(n+1)$ th antenna is the one that maximizes the channel capacity, that is,

$$
P_{n+1}^{\text {subopt }}=\underset{l \notin\left\{p_{1}^{\text {subopt }}, p_{2}^{\text {subopt }}, \cdots p_{n}^{\text {subopt }}\right\}}{\arg \max } C_{l}
$$

This process continues until all Q antennas are selected.

Also the same process can be implemented by deleting the antenna in descending order of decreasing channel capacity. Let $S_{n}$ denote a set of antenna indices in the nth iteration. In the initial step, we consider all 
antennas, $S_{l}=\left\{1,2, \cdots, N_{T}\right\}$, and select the antenna that contributes least to the capacity, that is,

$p_{1}^{\text {deleted }}=\arg \max \log _{2} \operatorname{det}\left(I_{N_{R}}+\frac{E_{X}}{Q N_{0}} H_{S_{1}-\left\{p_{1}\right\}} H_{S_{1}-\left\{p_{1}\right\}}^{H}\right)$

A good literature on exploitation of CSI for channel estimation and the types of antenna selection techniques can be found in [4-15].The antenna selected from above Equation will be deleted from the antenna index set, and there remaining antenna set is updated to $S_{2}=S_{1}-\left\{p_{1}^{\text {deleted }}\right\}$. If $\left|s_{2}\right|=N_{T}-1>Q$ we choose another antenna to delete. This will be the one that contributes least to the capacity now for the current antenna index set $\mathrm{S} 2$, that is,

$P_{2}^{\text {deleted }}=\operatorname{argmax} \log _{2} \operatorname{det}\left(I_{N_{R}}+\frac{E_{X}}{Q N_{0}} H_{S_{2}-\left\{p_{2}\right\}} H_{S_{2}-\left\{p_{2}\right\}}^{H}\right)$ Again, the remaining antenna index set is updated to $S_{3}=S_{2}-\left\{p_{2}^{\text {deleted }}\right\}$. This process will continue until all Q antennas are selected, that is, $\left|S_{n}\right|=Q$. The complexity of selection method in descending order is higher than that in ascending order.

From the performance perspective, however, the selection method in descending order outperforms that in ascending order when $1<\mathrm{Q}<\mathrm{N}_{\mathrm{T}}$. This is due to the fact that the selection method in descending order considers all correlations between the column vectors of the original channel gain before choosing the first antenna to delete.

When $\mathrm{Q}=1$, the selection method in descending order produces the same antenna index set as the optimal antenna selection method produces Equation (2). When $\mathrm{Q}=1$, however, the selection method in ascending order produces the same antenna index as the optimal antenna selection method in Equation (2) and achieves better performance than any other selection methods. In general, however, all these methods are just suboptimal, except for the above two special cases. Figure above shows the channel capacity with the selection method in descending order for various numbers of selected antennas with $\mathrm{N}_{T}=4$ and $\mathrm{N}_{\mathrm{R}}=4$. [6]

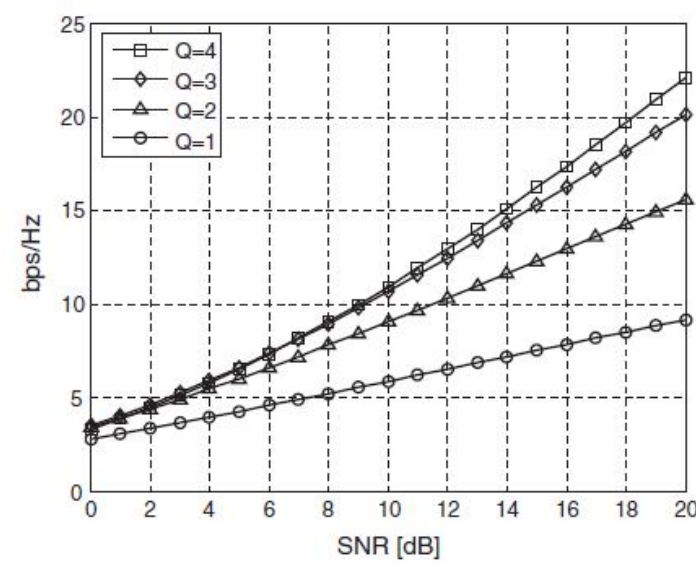

Figure 2: Channel capacities for antenna selection method in descending order.

\section{CONCLUSION}

In this paper, The Complexity-Reduced Antenna Selection Technique is discussed. As compared to the optimal antenna technique, complexity reduced antenna selection technique is better. Optimal antenna selection requires too much complexity depending on the total number of available transmit antennas. In order to reduce its complexity, a proposed a sub-optimal method is used. We adopted an approach in which additional antenna is selected in ascending order of increasing the channel capacity i.e., one antenna with the highest capacity is first selected we have used transmission techniques that can be used to exploit the CSI on the transmitter side. The CSI can be known completely or partially. Sometimes, only statistical information of the channel state is available. We have exploited such information for optimum antenna selection and hence for achieving the high channel capacity. Simulation results show that the channel capacity increases in proportion to the number of the selected antennas.

\section{REFERENCES}

[1] Menghui Yang, Tonghong Li, WeikangYang,Xin Su And Jing Wang (2009). A Channel Estimation Scheme for STBC-Based TDS-OFDM MIMO System. Eighth IEEE International Conference On Embedded Computing (2009). Page: 160-166.

[2] Dinesh B. Bhoyar, Dr. C. G. Dethe, Dr. M. M. Mushrif, Abhishek P. Narkhede (2013). Leaky Least Mean Square (Llms) Algorithm for Channel Estimation in BPSKQPSK-PSK MIMO-OFDM.System. International MultiConference on Automation, Computing, Communication, Control and Compressed Sensing, (2013). Page: 623.

[3] Jun Shikida, Satoshi Suyama, Hiroshi Suzuki, and Kazuhiko Fukawa (2010). Iterative Receiver Employing Multiuser Detection and Channel Estimation for MIMOOFDMA. IEEE 71st Vehicular Technology Conference (2010). Page: $1-5$ 
[4] MeikD"Orpinghaus, Adrian ISPAS and Heinrich Meyr (2011). Achievable Rate With Receivers Using Iterative Channel Estimation in Stationary Fading Channels. IEEE 8th International Symposium on Wireless Communication Systems, (2011). Page: 517-521.

[5] Bharath B. N. and Chandra R. Murthy. (2012) Channel Estimation At The Transmitter In a Reciprocal MIMO Spatial Multiplexing System. IEEE National Conference (2012), Page: 1-5.

[6] Reduced-Rank Estimation Of Non stationary TimeVariant Channels Using Subspace Selection. L. H. Xing, Zh. H. Yu, Zh. P. Gao, And L. Zha (2006)Channel Estimation For Transmitter Diversity OFDM Systems. $1^{\text {st }}$ IEEE Conference on Digital Object Identifier (2006). Page: $1-4$.

[7] JiaMeng, Wotao Yin, Yingying Li, Nam Tuan Nguyen, and Zhu Han (2012). IEEE Journal Of Selected Topics In Signal Processing, 6(1) February 2012. Page: 15-25.

[8] Thomas Zemen, and Andreas F. Molisch, (2012) Adaptive IEEE Transactions, 61(9) (2012). Page: 40424056

[9] Osama Ullah Khan, Shao-Yuan Chen, David D. Wentzloff, And Wayne E. Stark (2012). Impact of Compressed Sensing With Quantization On UWB Receivers With Multipath Channel Estimation. IEEE Journal on Emerging and Selected Topics In Circuits And Systems, 2(3), September 2012. Page: 460-469.

[10] Mihai-AlinBadiu, Carles Navarro Manch'On, and Bernard Henri Fleury (2013). Message-Passing Receiver Architecture with Reduced-Complexity Channel Estimation. IEEE Communications Letters, 17(7) (2013). Page: 1404-1407.

[11] ErenEraslan, BabakDaneshrad, and Chung-Yu Lou (2013). Performance Indicator For MIMO MMSE Receivers In The Presence of Channel Estimation Error. IEEE Wireless Communications Letters, 2(2), April 2013. Page: $211-214$.

[12] HarisGacanin (2013). Joint Iterative Channel Estimation and Guard Interval Selection of Adaptive Power line Communication Systems. IEEE 17th International Symposium On Power Line Communications And Its Applications (2013). Page: 197-202.

[13] Chao-Wei Huang, Tsung-Hui Chang, Xiangyun Zhou, and Y.-W. Peter Hong (2013). Two-Way Training For Discriminatory Channel Estimation in Wireless MIMO Systems. IEEE Transactions On Signal Processing, 61(10), May 15, 2013. Page: 2724-2738.

[14] Mohamed Marey, Moataz Samir, And Mohamed Hossam Ahmed (2013). Joint Estimation Of Transmitter And Receiver IQ Imbalance With Ml Detection For Alamouti OFDM Systems. IEEE Transactions on Vehicular Technology, 62(6), July 2013. Page: 28472853.

[15] Joham, M., Utschick,W., and Nossek, J.A., "Linear transmit processing in MIMO communications systems" , IEEE Transactions on Signal Processing, vol 53(8), 2005.Page: $2700-2712$. 